\title{
Technical
}

\section{Molecular Adsorbent Recirculating System (MARS) in Patients with Primary Nonfunction and Other Causes of Graft Dysfunction After Liver Transplantation in the Era of Extended Criteria Donor Organs}

\author{
R. Gaspari, F. Cavaliere, L. Sollazzi, V. Perilli, I. Melchionda, S. Agnes, A. Gasbarrini, and A.W. Avolio
}

\begin{abstract}
Liver dysfunction is an important cause of morbidity and mortality after orthotopic liver transplantation (OLT). The Molecular Adsorbent Recirculating System (MARS) is an albumin-based dialysis system designed to enhance the excretory function of a failing liver. MARS has been successfully used in patients affected by advanced liver disease and presenting with severe cholestasis. The aim of this study was to evaluate the safety and clinical efficacy of MARS in patients with liver dysfunction after OLT. Seven patients (primary nonfunction, 2 patients; graft dysfunction, 5 patients) fulfilled the inclusion criteria of serum bilirubin level $>15 \mathrm{mg} / \mathrm{dL}$ and least 1 of the following clinical signs: hepatic encephalopathy (HE) $\geq$ grade II, hepatorenal syndrome (HRS), and intractable pruritus. Graft and patient survival rates at 6 months were $42.8 \%$ and $57.1 \%$, respectively. All patients tolerated MARS treatment, with no adverse event. In all patients, a decrease in serum bilirubin $(P<.05)$, bile acids $(P<.05)$, serum creatinine, and ammonia levels was observed after treatment with MARS. A considerable improvement of HE, as well as renal and synthetic liver functions, was observed in 4 of 5 patients with graft dysfunction, but not among those with primary nonfunction. The patients with intractable pruritus showed significant improvement of this symptom after MARS therapy. Thus, MARS is a safe, therapeutic option for the treatment of liver dysfunction after OLT. Further studies are necessary to confirm whether this treatment is able to improve both graft and patient survival.
\end{abstract}

$\mathrm{L}$ IVER dysfunction is an important cause of morbidity and mortality after orthotopic liver transplantation (OLT). Liver dysfunction includes a most severe condition, namely primary nonfunction (PNF), and a less severe one, namely graft dysfunction (GD). PNF is characterized by the absence of graft function, leading in a few days to death if emergency retranplantation (re-OLT) is not performed. Conversely, GD is characterized by inadequate graft function, which may spontaneously recover, although over-
From the Department of Anaesthesia and Intensive Care (R.G., F.C., L.S., V.P., I.M.), Department of Surgery - Transplantation Service (S.A., A.W.A.), Department of Internal Medicine (A.G.), Catholic University of Rome, Italy.

Address reprint requests to Alfonso Wolfango Avolio, MD, Department of Surgery, Catholic University of Rome, L. go Gemelli 8 Rome 00168, Italy. E-mail: alfonso.avolio@rm.unicatt.it

0041-1345/09/\$-see front matter doi:10.1016/j.transproceed.2008.10.066 
whelming complications often occur. Patients with GD stay for a longer time in the intensive care unit (ICU), and experience longer hospitalizations, as well as worse recipient and graft survivals than patients without GD. ${ }^{1,2}$

The Molecular Adsorbent Recirculating System (MARS), an extracorporeal liver support device for patients with acute liver failure (ALF) or acute on chronic liver failure (AoCLF), replaces the detoxification function. MARS is a modified dialysis system whereby blood is dialyzed against an albumincontaining solution across a high-flux membrane. ${ }^{3}$ The technique allows removal of both albumin-bound (bilirubin and bile acids) and water-soluble (ammonia and urea) toxins that have accumulated in the blood of patients with liver failure. These molecules have been involved in the development of hepatic encephalopathy (HE), hepatorenal syndrome (HRS), hemodynamic instability, and progression to multiorgan failure (MOF). Several studies have shown that clearance of these substances by MARS leads to recovery from acute liver decompensation and/or provides a bridge to OLT. ${ }^{4-10}$ However, the role of MARS for patients with liver dysfunction after OLT has not yet been investigated. The aim of this study was to evaluate the safety and clinical efficacy of MARS treatment of patients with PNF and GD after OLT.

\section{PATIENTS AND METHODS}

From July 2001 to December 2007, 34 adult patients in the ICU affected by liver failure were treated with MARS (125 sessions). During this period, the 7 patients who presented with PNF or GD after their first OLT were enrolled into the study. The diagnosis of PNF was made on the basis of progressive HE, severe coagulopathy, transaminases $>3000 \mathrm{mU} / \mathrm{L}$, bilirubin levels $>10 \mathrm{mg} / \mathrm{mL}$, absence of bile production, and occurrence of renal, cardiovascular, and respiratory failure within the first $24-48$ hours after OLT. GD was defined as a less severe condition, characterized by a variable degree of graft dysfunction occurring at different times (ie, either early or late) after OLT. In our series there were 2 patients with PNF and 5 patients with GD. Causes of GD were as follows: sepsis from abdominal infection (case no. 1), severe chronic ductopenic allograft rejection (case no. 2), liver hypoperfusion due to hemorrhagic shock (case no. 4), multiple hepatic abscesses (case no. 5), and progressive cholestasis decompensation after a transfusion accident (case no. 7). The 2 patients with PNF were listed for urgent re-OLT, and only 1 patient with GD (case no. 5) was placed on the nonurgent waiting list for re-OLT due to multiple liver abscesses at 4 months after transplantation. Concomitant sepsis and renal failure was considered a contraindication for retransplantation.

Standard grafts were used in 2 cases and extended criteria donor (ECD) grafts $^{11,12}$ in 5 cases. For each case the extended criteria adopted are reported in Table 1.

In all patients, MARS treatment was performed in the absence of improvement in clinical and biochemical parameters obtained by standard medical therapy. Inclusion criteria were bilirubin level $>15 \mathrm{mg} / \mathrm{dL}$, without the evidence of an extraepatic origin, and at least 1 of the following clinical signs: HE $\geq$ grade II, HRS, and intractable pruritus. HE was assessed according to West Haven Criteria. ${ }^{13}$ The intensity of pruritus was evaluated by a visual analogic scale (VAS) ranging from 0 (no pruritus) to 10 (unbearable pruritus). ${ }^{14}$ The median time between OLT and the first MARS treatment was 9 days (range, 2-180). The Model for

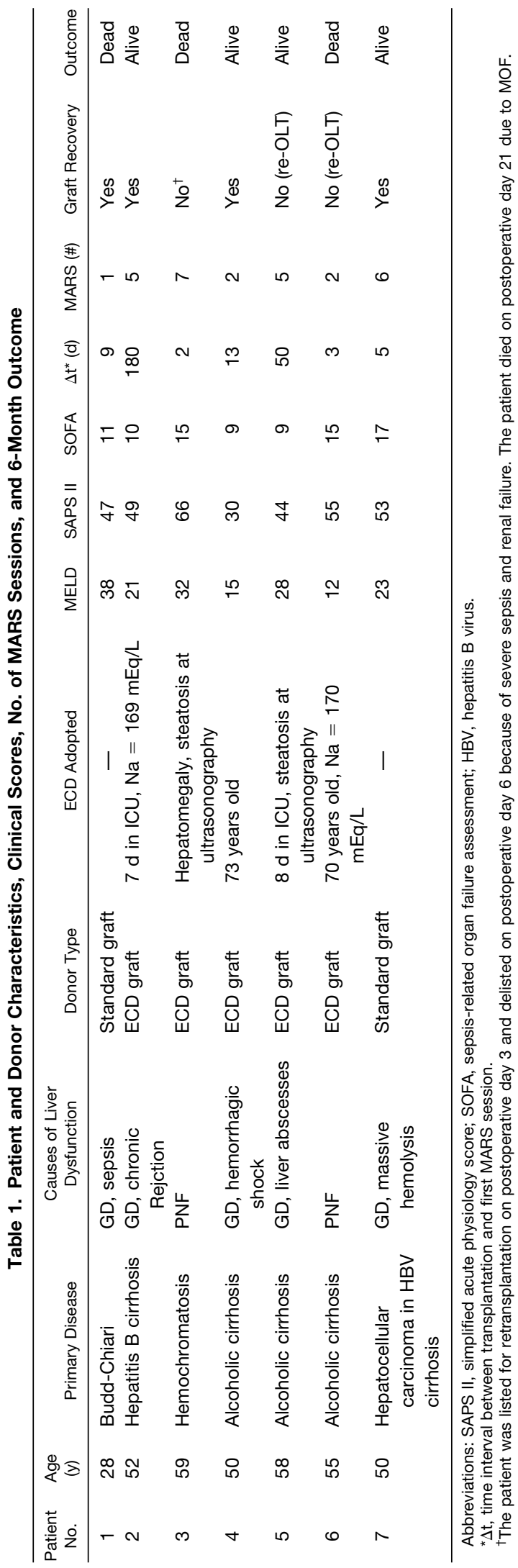


End-Stage Liver Disease (MELD) was calculated at hospital admission; the Simplified Acute Physiology Score (SAPS II) and the Sepsis-Related Organ Failure Assessment (SOFA) were calculated at the beginning of MARS therapy.

The patients with HE grade $\geq$ III were intubated and mechanically ventilated (Servo 300, Siemens, Copenhagen, Denmark). One patient was mechanically ventilated due to severe hypoxia. For ventilation and control of agitation, we used sedation with continuous infusion of propofol and remifentanil. Renal support therapy was provided by continuous veno-venous hemofiltration (PrismaHospal, Bologna, Italy). Two patients with GD suffered from intractable pruritus despite standard medical treatment, such as cholestyramine, ursodeoxycholic acid, and naloxone. Standard monitoring included a peripheral arterial line and a central venous catheter. During the MARS treatment, we continuously recorded cardiovascular parameters, arterial oxygen saturation, and body temperature. Standard liver tests, ammonia, bile acids, coagulation profile, renal function tests, electrolyte balance, complete blood cell count, and arterial blood gas analysis were performed before and after each MARS treatment.

The patients were followed for 180 days after the discontinuation of MARS therapy. The 6-month outcomes were evaluated as spontaneous graft recovery, death, or bridge to re-OLT.

The study, which was approved by the ethics committee, was performed after written consent obtained from each patient or next of kin.

\section{MARS Dialysis Therapy}

MARS treatment was performed through a standard dialysis catheter placed into the internal jugular or femoral vein. The MARS device (Gambro AB, Lund, Sweden) was connected to a standard dialysis machine (Integra-Hospal, Modena, Italy) or to a hemofiltration device (Prisma-Hospal, Modena, Italy). A continuous infusion of heparin sulfate was used to prevent thrombosis in the extracorporeal circuit, the dosage was adjusted to maintain an activated clotting time between 150 and 180 seconds. Antithrombin III was administered to maintain a serum level $>70 \%$. MARS treatment was performed every other day (or daily) until the patient showed an improvement in graft function namely, spontaneous reduction of serum bilirubin levels for 3 consecutive days without treatments.

\section{Statistical Analysis}

Data are expressed as mean values $\pm \mathrm{SD}$ or as medians and ranges, when appropriate. Differences between pre-MARS and postMARS values were assessed using analysis of variance (ANOVA) one-way test. A $P$ value $\leq .05$ was considered significant.

\section{RESULTS}

Twenty-eight MARS sessions were performed in 7 patients ( 5 males and 2 females) of median age 52 years (range, 28-59). Each patient underwent 1 to 7 MARS sessions, lasting $5 \pm 1$ hours. Demographic data, primary liver disease, causes of liver dysfunction after OLT, donor quality (standard graft vs ECD graft), clinical score, and number of MARS sessions are reported in Table 1.

\section{Safety and Tolerability}

MARS therapy was well tolerated in all patients; no bleeding complications were observed. The median platelet count decreased after each treatment session, but the reduction was not statistically significant.

\section{Biochemical and Clinical Parameters}

After each MARS treatment we observed a reduction in bilirubin (from $40.0 \pm 11.3$ to $29.5 \pm 7.3 \mathrm{mg} / \mathrm{dL}$ ), bile acids (from $131 \pm 73$ to $81 \pm 38 \mu \mathrm{mol} / \mathrm{L}$ ), creatinine (from $2.2 \pm$ 1.2 to $2.0 \pm 1.5 \mathrm{mg} / \mathrm{dL}$ ), and ammonia levels (from $152 \pm$ 177 to $143 \pm 179$ gamma/dL). Differences were significant only for bilirubin and bile acids levels $(P<.05)$. Clinical effectiveness was assessed by the improvement of HE, HRS, pruritus, and liver synthetic function. An improvement in HE grade (defined as reduction $\geq 1$ grade from baseline) was observed in all patients with GD, but not in those with PNF. In 1 patient with GD (case no. 1), the significant reduction of $\mathrm{HE}$ allowed discontinuation of mechanical ventilation. With regard to renal function, there was no improvement in patients affected by HRS ( 2 patients with PNF), whereas those with less severe renal damage displayed good renal functional recovery, as assessed by increased creatinine clearance. After each treatment we also observed a significant reduction in symptoms in 2 patients (case no. 2 and no. 5) presenting with intractable pruritus. In 1 patient (case no. 2) the pruritus disappeared completely after 5 MARS sessions (VAS decreased from 10 to 0 ), allowing discontinuation of symptomatic therapy; in the other patient pruritus decreased, but did not disappear (VAS decreased from 10 to 4). Considering liver function, only 4 of 5 patients with GD showed recovery of hepatic function: reduction in prothrombin time and increase in serum glucose and albumin levels. Conversely, no change in synthetic liver function was observed in patients with PNF (case no. 3 and no. 6) and in the patient with GD due to liver abscesses (case no. 5). Finally, in the patient with severe cholestasis due to massive hemolysis (case no. 7), an unexpected improvement of respiratory function with a strong increase in arterial oxygen pressure (from 55 to 256 $\mathrm{mm} \mathrm{Hg}$ ) was observed after the first MARS treatment.

\section{Survival}

Graft and patient survival rates at 6 months were $42.8 \%$ and $57.1 \%$, respectively. None of the patients with PNF survived, whereas 4 of 5 patients with GD survived at the end of the follow-up (Table 1). One patient with PNF (case no. 3 ) died due to severe sepsis and MOF 21 days after the first of 7 MARS sessions. The other PNF patient (case no. 6) underwent a re-OLT on postoperative day 4 after 2 MARS treatments. Unfortunately, this patient developed severe brain damage, and died due to pneumonia 150 days thereafter, despite good liver function. Three of 5 patients with GD who fully recovered graft function were alive and well after a 6-month follow-up. One patient (case no. 1) showed a good recovery after MARS treatment, but died 91 days thereafter, due to sepsis. The remaining GD patient (case no. 5) underwent retransplantation after 5 MARS sessions; he is alive and well at the end of the 6 months of follow-up. 


\section{DISCUSSION}

PNF was a critical complication in the early experience of liver transplantation, accounting for a high morbidity of the graft and a high mortality of the patient. This condition, when not treated by prompt re-OLT, led to death within a few days. ${ }^{1}$ Several risk factors have been identified as predictors of PNF, such as inadequate ICU donor management, primordial perfusion solutions, and long cold ischemia times. In the late $1980 \mathrm{~s}$, the introduction of the University of Wisconsin solution reduced the rate of PNF to $<5 \%$. In contrast to PNF, GD occurs among a larger number of patients. Although some patients with GD recover spontaneously by adopting conservative measures, worse recipient and graft survival rates have been recorded among recipients with GD than those without it. ${ }^{1,2} \mathrm{Re}$ cently, as organ acceptance criteria have changed, and the use of so-called ECD grafts ${ }^{11,12}$ has increased, the risk for development of liver dysfunction after OLT has increased again. Although some ECD do not affect posttransplantation recovery, as is the case for a donor with serum positivity for anti-HBc or one with an increased level of prostatespecific antigens, other criteria, such as elderly age or steatosis, ${ }^{15}$ have been associated with a higher prevalence of PNF and with reduced graft and patient survival rates. However, graft recovery needs time. Meanwhile, the longer stay in hospital and in the ICU increase the risk of nosocomial (such as pulmonary) infections, and of other complications, such as renal failure and rejection. The treatment of an infection often requires reduction of im- munosuppressive drugs with an increased risk of rejection. For these reasons, the pattern of liver damage after OLT has moved from PNF (which still exists, yet with a lower prevalence than before) to a more extensive condition, namely graft dysfunction, which includes all cases of apparently reversible impairment.

In recent years, a new category of therapeutic procedures has been introduced to support liver function in patients with advanced liver disease. Liver support devices, which are classified as bio-artificial systems and artificial devices, bridge patients suffering from ALF and/or from AoCLF, either to recovery or to transplantation. The bio-artificial systems, which contain a cellular line of various origins (either human or porcine) placed on an artificial structure, were developed to replace liver functions. Besides the technical difficulties, no ultimate evidence has supported the benefit of these devices. ${ }^{16}$ The nonbiological or artificial devices do not have metabolic activity. They were designed to enhance the excretory function of the liver. In patients with liver failure, they counter the accumulation of circulating toxins. According to the "albuminbound toxin hypothesis," these substances have been implicated in the development and maintenance of HE, HRS, hemodynamic instability, and MOF.

MARS, first introduced into clinical practice in $1999,{ }^{4}$ is the extracorporeal detoxification device more commonly used throughout the world. MARS is a simple and safe, albumin-based dialysis system that allows the removal of both albumin-bound and water-soluble toxins in the lowmolecular and middle-molecular weight ranges. Several

Table 2. A Synthesis of Clinical Experiences With MARS in Liver Dysfunction After OLT

\begin{tabular}{|c|c|c|c|c|c|c|}
\hline Author & Year & $\begin{array}{l}\text { Total No. of } \\
\text { Patients }\end{array}$ & $\begin{array}{l}\text { No. of Cases } \\
\text { With Liver } \\
\text { Dysfunction }\end{array}$ & $\begin{array}{l}\text { Indications for MARS in Liver Dysfunction } \\
\text { After OLT (No. of Patients) }\end{array}$ & Follow-Up (mo) & Outcome \\
\hline Loock et $\mathrm{al}^{18}$ & 2001 & 4 & 4 & Poor graft function after split liver (4) & - & $\begin{array}{l}\text { Alive } 3 \text { (1 re-OLT), } \\
\text { dead } 1\end{array}$ \\
\hline Jost et $\mathrm{al}^{19}$ & 2001 & 11 & 1 & PNF (1) & - & $?$ \\
\hline Kasakow et $\mathrm{al}^{20}$ & 2001 & 1 & 1 & Early GD after split liver (1) & - & Alive re-OLT \\
\hline Delafosse et $\mathrm{al}^{21}$ & 2001 & 5 & 2 & Acute severe rejection (2) & - & $\begin{array}{l}\text { Alive } 1 \text { (re-OLT), } \\
\text { dead } 1\end{array}$ \\
\hline Kellersmann et $\mathrm{al}^{22}$ & 2002 & 5 & 3 & $\begin{array}{l}\text { Portal venous thrombosis (1), infection } \\
\text { (1), PNF (1) }\end{array}$ & - & $\begin{array}{l}\text { Alive } 1, \text { dead } 2 \text { (1 } \\
\text { re-OLT) }\end{array}$ \\
\hline Bellmann et $\mathrm{al}^{23}$ & 2004 & 7 & 7 & Pruritus: cholestatic graft failure (7) & 12 & $\begin{array}{l}\text { Alive } 6(3 / 6 \text { re-OLT), } \\
\text { dead } 1\end{array}$ \\
\hline Novelli et $\mathrm{al}^{6}$ & 2005 & 116 & 30 & PNF (13), delayed nonfunction (17) & - & $\begin{array}{l}\text { Alive } 23 \text { (8 re-OLT), } \\
\text { dead } 7 \text { (3 re-OLT) }\end{array}$ \\
\hline Koivusalo et $\mathrm{al}^{7}$ & 2005 & 101 & 10 & $\begin{array}{l}\text { PNF (2), primary dysfunction (5), late } \\
\text { graft failures (3) }\end{array}$ & 6-36 (mean, 16) & $\begin{array}{l}\text { Alive } 8 \text { ( } 3 \text { re-OLT), } \\
\text { dead } 2\end{array}$ \\
\hline Camus et $\mathrm{al}^{8}$ & 2006 & 23 & 5 & $\begin{array}{l}\text { Hepatic artery thrombosis (2), acute } \\
\text { rejection (2), PNF (1) }\end{array}$ & 6 & $?$ \\
\hline Montero et $\mathrm{al}^{24}$ & 2006 & 4 & 3 & $\begin{array}{l}\text { Pruritus: ductopenic graft rejection (2), } \\
\text { HCV recurrence (1) }\end{array}$ & $12-24$ & Alive 3 (re-OLT 1) \\
\hline Hetz et $a^{25}$ & 2006 & 12 & 12 & Early GD (12) & 12 & Alive 8 , dead 4 \\
\hline Scheingraber et $\mathrm{al}^{26}$ & 2007 & 19 & 6 & PNF (2), GD (4) & & Alive ?, dead? \\
\hline Gaspari et $\mathrm{al}^{9}$ & 2006 & 28 & 6 & PNF (2), primary dysfunction (4) & 6 & Alive 4 , dead 2 \\
\hline Chiu et al $^{10}$ & 2006 & 22 & 4 & $\begin{array}{l}\text { Acute graft rejection (2), } \mathrm{HCV} \\
\text { recurrence (1), biliary Anastomosis } \\
\text { narrowing (1) }\end{array}$ & - & Dead 4 (re-OLT 2) \\
\hline
\end{tabular}


studies have demonstrated that the clearance of these molecules by MARS leads to recovery from acute liver decompensation and/or provides a bridge to OLT among patients with ALF and AoCLF. Most articles have shown a beneficial effect of MARS on HE, HRS, and short-term patient survival. ${ }^{5-10,17}$ To date, more than 6500 patients have been treated worldwide. The most common indications have been ALF and AoCLF, ${ }^{5-10,15}$ whereas in patients with liver dysfunction after OLT, MARS has rarely been used. In addition, the patients with liver dysfunction treated with MARS are reported to be a minority of those who took part in larger experiences with MARS (Table 2). The indications for MARS after OLT were PNF, GD (either early or late), and severe pruritus. ${ }^{6-10,18-26}$ However, the definition of graft failure, the length of follow-up, the concomitant use of the retransplantation as a rescue procedure, and the outcomes have often not been specified.

Only a preliminary prospective study on a homogeneous group of 12 patients who met inclusion criteria for early GD has been reported. In that study, patients with PNF were not included. The authors reported a 12-month survival rate of $66 \% .^{25}$ In another recent study on patients with liver failure, including OLT patients, the authors, using the disappearance rate of indocyanine green (ICG), described an improvement in plasma disappearance rates and in clinical conditions after MARS treatment only among patients with GD, but not those with PNF. ${ }^{26}$ The largest experience with MARS in transplant recipients refers to 30 subjects: 13 PNF and 17 delayed nonfunction. ${ }^{6}$ That article contains a MARS group of 116 patients, but failed to clearly define GD; further, the follow-up and outcomes are not specifically reported.

In the present experience MARS was used as a bridge to spontaneous recovery or retransplantation. However, 2 patients with PNF were listed for retransplantation, using MARS as a supportive not a substitutive therapy. Criteria for retransplantation have not been well codified. Although data support the indication for several causes of graft failure (biliary abscesses, hepatitis $\mathrm{C}$ virus [HCV] recurrence, and chronic rejection), the indications for retransplantation after PNF have not been completely defined. However, in the largest survey concerning factors that affect the outcome after liver retransplantation, recipient age and level of creatinine were associated with the poorest outcomes. ${ }^{27}$ Although the 2 cases with PNF were listed for retransplantation, in 1 case the retransplantation was not performed because of severe sepsis and renal failure.

Our data suggested that MARS is beneficial only in patients with GD after OLT. With regard to GD, graft function fully recovered in 3 of 5 patients, 1 patient recovered from GD, but died because of sepsis; the remaining patient was successfully bridged to re-OLT. Therefore, only in patients with GD did the clearance of albuminbound and water-soluble toxins obtained with MARS lead to improvement of HE, renal function, pruritus, and synthetic liver function. However, the limited experience and the absence of control group are limitations to this observation.

In conclusion, in our experience MARS represented an effective treatment to support patients with GD. In this subgroup of patients the use of MARS, together with standard intensive care measures, leads to the management of progressive bilirubin increases, and allowed support of liver, neurological, and renal functions. Moreover, MARS appeared to be capable of improving survival rates. Multicenter, randomized, controlled studies, comparing MARS with other extracorporeal treatment modalities and/or standard medical therapy, should better define the indications and drawbacks of this approach.

\section{REFERENCES}

1. Ploeg RJ, D'Alessandro AM, Knechtle SJ, et al: Risk factor for primary dysfunction after liver transplantation: a multivariate analysis. Transplantation 55:807, 1993

2. Deschênes M, Belle SH, Krom RA, et al: Early allograft dysfunction after liver transplantation: a definition and predictors of outcome. National Institute of Diabetes and Digestive and Kidney Diseases Liver Transplantation Database. Transplantation 66:302, 1998

3. Stange J, Ramlow W, Mitzner S, et al: Dialysis against a recycled albumin solution enables the removal of albumin-bound toxins. Artif Organs 17:809, 1993

4. Stange J, Mitzner SR, Risler T, et al: Molecular adsorbents recycling system (MARS): clinical result of a new membrane-based blood purification system for bioartificial liver support. Artif Organs 23:319, 1999

5. Heemann U, Treichel U, Loock J, et al: Albumin dialysis in cirrhosis with superimposed acute liver injury: a prospective, controlled study. Hepatology 36:949, 2002

6. Novelli G, Rossi M, Pretagostini R, et al: One hundred sixteen cases of acute liver failure treated with MARS. Transplant Proc 37:2557, 2005

7. Koivusalo AM, Vakkuri A, Höckerstedt K, et al: Experience of MARS therapy with and without transplantation in 101 patients with liver insufficiency. Transplant Proc 37:3315, 2005

8. Camus C, Lavoué S, Gacouin A, et al: Molecular adsorbent recirculating system dialysis in patients with acute liver failure who are assessed for liver transplantation. Intensive Care Med 32:1817, 2006

9. Gaspari R, Avolio AW, Zileri Dal Verme L, et al: Molecular adsorbent recirculating system in liver transplantation: safety and efficacy. Transplant Proc 38:3544, 2006

10. Chiu A, Chan LM, Fan ST: Molecular adsorbent recirculating system treatment for patients with liver failure: the Hong Kong experience. Liver Int 26:695, 2006

11. Tector AJ, Mangus RS, Chestovich P, et al: Use of extended criteria livers decreases wait time for liver transplantation without adversely impacting posttransplant survival. Ann Surg 244:439, 2006

12. Renz JF, Kin C, Kinkhabwala M, et al: Utilization of extended donor criteria liver allografts maximizes donor use and patient access to liver transplantation. Ann Surg 242:556, 2005

13. Conn HO, Leevy CM, Vlahcevic ZR, et al: Comparision of lactulose and neomycin in the treatment of chronic portal-systemic encephalopathy. A double blind controlled trial. Gastroenterology 72:573, 1977

14. Müller C, Pongratz S, Pidlich J, et al: Treatment of pruritus in chronic liver disease with the 5-hydroxytryptamine receptor type 3 antagonist ondansetron: a randomized, placebo-controlled, doubleblind cross-over trial. Eur J Gastroenterol Hepatol 10:865, 1998

15. Briceno J, Padillo J, Rufián S, et al: Assignment of steatotic liver by the Mayo model for end stage liver disease. Transpl Int 18:577, 2005 
16. Demetriou AA, Brown RS, Busuttil RW, et al: Prospective, randomized, multicenter, controlled trial of a bioartificial liver in treating acute liver failure. Ann Surg 239:660, 2004

17. Hassanein TI, Tofteng F, Brown RS Jr, et al: Randomized controlled study of extracorporeal albumin dialysis for hepatic encephalopathy in advanced cirrhosis. Hepatology 46:1853, 2007

18. Loock J, Treichel U, Gerken G, et al: Treatment of splitliver recipients with poor graft function by albumin-dialysis (Mars). Z Gastroenterol 39(suppl 2):18, 2001

19. Jost U, Schreiter D, Sceibner L, et al: Continuos venovenous hemofiltration with extracorporeal albumin dialysis "MARS" in critically ill patients before and after liver transplantation. Z Gastroenterol 39(suppl 2):43, 2001

20. Kasakow L, Hommannn M, Wagner TH, et al: Application of artificial liver support (MARS) after split-liver transplantation in an infant. Z Gastroenterol 39(suppl 2):50, 2001

21. Delafosse B, Garnier E, Durmortier J, et al: Experience with the MARS in 5 patients experiencing hepatic failure. Z Gastroenterol 39(suppl 2):50, 2001
22. Kellersmann R, Gassel HJ, Bühler C, et al: Application of molecular adsorbent recirculating system in patients with severe liver failure after hepatic resection or transplantation: initial singlecentre experiences. Liver 22(suppl 2):56, 2002

23. Bellmann R, Graziadei IW, Feistritzer C, et al: Treatment of refractory cholestatic pruritus after liver transplantation with albumin dialysis. Liver Transpl 10:107, 2004

24. Montero JL, Pozo JC, Barrera P, et al: Treatment of refractory cholestatic pruritus with molecular adsorbent recirculating system (MARS). Transplant Proc 38:2511, 2006

25. Hetz H, Faybik P, Berlakovich G, et al: Molecular adsorbent recirculating system in patients with early allograft dysfunction after liver transplantation: a pilot study. Liver Transpl 12:1357, 2006

26. Scheingraber S, Richter S, Igna D, et al: Indocynine green elimination but not bilirubin indicates improvement of graft function during MARS therapy. Clin Transplant 21:689, 2007

27. Azoulay D, Linhares MM, Huguet E, et al: Decision for retransplantation of the liver: an experience-and cost-based analysis. Ann Surg 236:713, 2002 